Int. Journal of Math. Analysis, Vol. 7, 2013, no. 2, 85 - 89

\title{
Boundary Set on Bigeneralized Topological Spaces
}

\author{
Supunnee Sompong and Sa-at Muangchan \\ Department of Mathematics and Statistics \\ Faculty of Science and Technology \\ Sakon Nakhon Rajabhat University \\ Sakon Nakhon 47000, Thailand \\ s_sanpinij@yahoo.com, msaat@hotmail.com
}

\begin{abstract}
The purpose of this paper is to introduce the concept and some fundamental properties of boundary set on bigeneralized topological spaces.
\end{abstract}

Keywords: generalized topological space, bigeneralized topological space, boundary

\section{Introduction}

In 1963, J.C. Kelly [1] introduced the notion of bitopological spaces and extended some of the standard results of separation axioms in a topological space to a bitopological space. The concepts of generalized topologies or briefly $G T$ was introduced by Á. Császár [2] in 2002. He also introduced two kinds of generalized continuity. In particular, generalized continuity admits a characterization furnishing a known characterization of $\theta$-continuous maps. In 2010, C. Boonpok [4] introduced the notion of bigeneralized topological spaces and the concepts of weakly functions. He also investigated some of their characterizations.

The purpose of this paper is to introduce the concept of boundary set on bigeneralized topological spaces and some of their simple properties.

\section{Preliminaries}

Let $X$ be a nonempty set and $g$ be a collection of subsets of $X$. Then $g$ is called a generalized topology (briefly $G T$ ) on $X$ if $\emptyset \in g$ and if $G_{i} \in g$ for $i \in I \neq \emptyset$ then $G=\bigcup_{i \in I} G_{i} \in g$.

By $(X, g)$, we denote a nonempty set $X$ with a generalized topology $g$ on $X$ and it is called a generalized topological space (briefly GTS) on $X$. The 
elements of $G$ are called $g$-open sets and the complements are called $g$-closed sets.

Theorem 2.1. [2] Let $(X, g)$ be a generalized topological space. Then

1. $C l(A)=X \backslash \operatorname{Int}(X \backslash A)$;

2. $\operatorname{Int}(A)=X \backslash C l(X \backslash A)$.

Proposition 2.2. [3] Let $(X, g)$ be a generalized topological space. For subsets $A$ and $B$ of $X$, the following properties holds;

1. $C l(X \backslash A)=X \backslash \operatorname{Int}(A)$ and $\operatorname{Int}(X \backslash A)=X \backslash C l(A)$;

2. If $X \backslash A \in g$, then $C l(A)=A$ and if $A \in g$, then $\operatorname{Int}(A)=A$;

3. If $A \subseteq B$, then $C l(A) \subseteq C l(B)$ and $\operatorname{Int}(A) \subseteq \operatorname{Int}(B)$;

4. $A \subseteq C l(A)$ and $\operatorname{Int}(A) \subseteq A$;

5. $C l(C l(A))=C l(A)$ and $\operatorname{Int}(\operatorname{Int}(A))=\operatorname{Int}(A)$.

Next, we recall some notions and notations of bigeneralized topological spaces.

Definition 2.3. [4] Let $X$ be a nonempty set and $g_{X}^{1}, g_{X}^{2}$ be generalized topologies on $X$. A triple $\left(X, g_{X}^{1}, g_{X}^{2}\right)$ is called a bigeneralized topological space (briefly $B G T S$ ).

Definition 2.4. [4] A subset $A$ of a bigeneralized topological space $\left(X, g_{X}^{1}, g_{X}^{2}\right)$ is called $g_{X}^{i} g_{X}^{j}-$ closed if $A=g^{i} C l\left(g^{j} C l(A)\right)$, where $i, j=1$ or 2 and $i \neq j$. The complement of $g_{X}^{i} g_{X}^{j}-$ closed set is called $g_{X}^{i} g_{X}^{j}-$ open.

Proposition 2.5. [4] Let $\left(X, g_{X}^{1}, g_{X}^{2}\right)$ be a bigeneralized topological space. Then $A$ is $g_{X}^{i} g_{X}^{j}$-open subset of $\left(X, g_{X}^{1}, g_{X}^{2}\right)$ if and only if $A=g^{i} \operatorname{Int}\left(g^{j} \operatorname{Int}(A)\right)$.

\section{Boundary}

In this section, we introduce the concept and study some fundamental properties of boundary set on bigeneralized topological spaces.

Definition 3.1. Let $\left(X, g_{X}^{1}, g_{X}^{2}\right)$ be a bigeneralized topological space, $A$ be a subset of $X$ and $x \in X$. We called $x$ is $(i, j)-g_{X}$ - boundary point of $A$ if $x \in g^{i} C l\left(g^{j} C l(A)\right) \cap g^{i} C l\left(g^{j} C l(X \backslash A)\right)$. We denote the set of all $(i, j)-$ $g_{X}$-boundary point of $A$ by $g B d r_{i j}(A)$ where $i, j=1,2$ and $i \neq j$.

From definition we have $g B d r_{i j}(A)=g^{i} C l\left(g^{j} C l(A)\right) \cap g^{i} C l\left(g^{j} C l(X \backslash A)\right)$.

Example 3.2. Let $X=\{1,2,3,4\}$. Define two generalized topologies $g_{X}^{1}$ and $g_{X}^{2}$ on $X$ as follows: $g_{X}^{1}=\{\emptyset,\{1,2\},\{3\},\{1,2,3\}\}$ and $g_{X}^{2}=\{\emptyset,\{1\},\{2,3\},\{1,2,3\}\}$.

Hence, $g B d r_{12}(\{3\})=X, g B d r_{21}(\{3\})=\{2,3,4\}$ and

$$
g B d r_{12}(\{2\})=X=g B d r_{21}(\{2\}) .
$$


Lemma 3.3. Let $\left(X, g_{X}^{1}, g_{X}^{2}\right)$ be a bigeneralized topological space and $A$ be a subset of $X$. Then $g B d r_{i j}(A)=g B d r_{i j}(X \backslash A)$ where $i, j=1,2$ and $i \neq j$.

Proof. $g B d r_{i j}(A)=g^{i} C l\left(g^{j} C l(A)\right) \cap g^{i} C l\left(g^{j} C l(X \backslash A)\right)$

$$
\begin{aligned}
& =g^{i} C l\left(g^{j} C l(X \backslash(X \backslash A))\right) \cap g^{i} C l\left(g^{j} C l(X \backslash A)\right) \\
& =g B d r_{i j}(X \backslash A)
\end{aligned}
$$

Thus $g B d r_{i j}(A)=g B d r_{i j}(X \backslash A)$ where $i, j=1,2$ and $i \neq j$.

Theorem 3.4. Let $\left(X, g_{X}^{1}, g_{X}^{2}\right)$ be a bigeneralized topological space and $A, B$ be a subset of $X$. Then for any $i, j=1,2$ and $i \neq j$, we have the following statements;

1. $g B d r_{i j}(A)=g^{i} C l\left(g^{j} C l(A)\right) \backslash g^{i} \operatorname{Int}\left(g^{j} \operatorname{Int}(A)\right)$;

2. $g B d r_{i j}(A) \cap g^{i} \operatorname{Int}\left(g^{j} \operatorname{Int}(A)\right)=\emptyset$;

3. $g B d r_{i j}(A) \cap g^{i} \operatorname{Int}\left(g^{j} \operatorname{Int}(X \backslash A)\right)=\emptyset$;

4. $g^{i} C l\left(g^{j} C l(A)\right)=g B d r_{i j}(A) \cup g^{i} \operatorname{Int}\left(g^{j} \operatorname{Int}(A)\right)$;

5. $X=g^{i} \operatorname{Int}\left(g^{j} \operatorname{Int}(X \backslash A)\right) \cup g B d r_{i j}(A) \cup g^{i} \operatorname{Int}\left(g^{j} \operatorname{Int}(A)\right)$

is a pairwise disjoint union.

Proof. Assume that $\left(X, g_{X}^{1}, g_{X}^{2}\right)$ is a bigeneralized topological space and $A, B$ are subsets of $X$.

1. $g B d r_{i j}(A)=g^{i} C l\left(g^{j} C l(A)\right) \cap g^{i} C l\left(g^{j} C l(X \backslash A)\right)$

$$
\begin{aligned}
& =g^{i} C l\left(g^{j} C l(A)\right) \cap\left[X \backslash g^{i} \operatorname{Int}\left(g^{j} \operatorname{Int}(A)\right)\right] \\
& =g^{i} C l\left(g^{j} C l(A)\right) \backslash g^{i} \operatorname{Int}\left(g^{j} \operatorname{Int}(A)\right) .
\end{aligned}
$$

2. From (1), it's obvious that, $g B d r_{i j}(A) \cap g^{i} \operatorname{Int}\left(g^{j} \operatorname{Int}(A)\right)=\emptyset$.

3. By Lemma 3.3 and (2), we have $g B d r_{i j}(A) \cap g^{i} \operatorname{Int}\left(g^{j} \operatorname{Int}(X \backslash A)\right)=\emptyset$.

4. $g^{i} C l\left(g^{j} C l(A)\right)=\left[g^{i} C l\left(g^{j} C l(A)\right) \backslash g^{i} \operatorname{Int}\left(g^{j} \operatorname{Int}(A)\right)\right] \cup g^{i} \operatorname{Int}\left(g^{j} \operatorname{Int}(A)\right)$

$$
=g B d r_{i j}(A) \cup g^{i} \operatorname{Int}\left(g^{j} \operatorname{Int}(A)\right) \text {. }
$$

5. $g^{i} \operatorname{Int}\left(g^{j} \operatorname{Int}(X \backslash A)\right) \cup g B d r_{i j}(A) \cup g^{i} \operatorname{Int}\left(g^{j} \operatorname{Int}(A)\right)$

$$
\begin{aligned}
& =\left[X \backslash g^{i} C l\left(g^{j} C l(A)\right)\right] \cup g^{i} C l\left(g^{j} C l(A)\right) \\
& =X .
\end{aligned}
$$

By (2) and (3) we have $g B d r_{i j}(A) \cap g^{i} \operatorname{Int}\left(g^{j} \operatorname{Int}(A)\right)=\emptyset$

and $g B d r_{i j}(A) \cap g^{i} \operatorname{Int}\left(g^{j} \operatorname{Int}(X \backslash A)\right)=\emptyset$.

Now, we will show that $g^{i} \operatorname{Int}\left(g^{j} \operatorname{Int}(A)\right) \cap g^{i} \operatorname{Int}\left(g^{j} \operatorname{Int}(X \backslash A)\right)=\emptyset$.

Since $g^{i} \operatorname{Int}\left(g^{j} \operatorname{Int}(A)\right) \cap g^{i} \operatorname{Int}\left(g^{j} \operatorname{Int}(X \backslash A)\right) \subseteq A \cap(X \backslash A)=\emptyset$. we also have $g^{i} \operatorname{Int}\left(g^{j} \operatorname{Int}(A)\right) \cap g^{i} \operatorname{Int}\left(g^{j} \operatorname{Int}(X \backslash A)\right)=\emptyset$.

Therefore, $X=g^{i} \operatorname{Int}\left(g^{j} \operatorname{Int}(A)\right) \cup g B d r_{i j}(A) \cup g^{i} \operatorname{Int}\left(g^{j} \operatorname{Int}(X \backslash A)\right)$

is a pairwise disjoint union. 
Theorem 3.5. Let $\left(X, g_{X}^{1}, g_{X}^{2}\right)$ be a bigeneralized topological space and $A$ be a subset of $X$. Then for any $i, j=1,2$ and $i \neq j$, we have;

1. A is $g_{X}^{i} g_{X}^{j}-$ closed if and only if $g B d r_{i j}(A) \subseteq A$.

2. $A$ is $g_{X}^{i} g_{X}^{j}-$ open if and only if $g B d r_{i j}(A) \subseteq(X \backslash A)$.

Proof. Let $A$ be a subset of $X$.

1. ( $\Longrightarrow)$ Assume that $A$ is $g_{X}^{i} g_{X}^{j}$-closed. Thus $g^{i} C l\left(g^{j} C l(A)\right)=A$.

$g B d r_{i j}(A) \cap(X \backslash A)=g^{i} C l\left(g^{j} C l(A)\right) \cap g^{i} C l\left(g^{j} C l(X \backslash A)\right) \cap(X \backslash A)$ $=A \cap g^{i} C l\left(g^{j} C l(X \backslash A)\right) \cap(X \backslash A)=\emptyset$.

Therefore, $g B d r_{i j}(A) \subseteq A$.

$(\Longleftarrow)$ Assume that $g B d r_{i j}(A) \subseteq A$. Thus $g B d r_{i j}(A) \cap(X \backslash A)=\emptyset$, and also $g^{i} C l\left(g^{j} C l(A)\right) \cap(X \backslash A)=g^{i} C l\left(g^{j} C l(A)\right) \cap\left[g^{i} C l\left(g^{j} C l(X \backslash A)\right) \cap(X \backslash A)\right]$

$=g B d r_{i j}(A) \cap(X \backslash A)$ $=\emptyset$.

Therefore, $g^{i} C l\left(g^{j} C l(A)\right) \subseteq A$. But $A \subseteq g^{i} C l\left(g^{j} C l(A)\right)$.

Consequently, $A=g^{i} C l\left(g^{j} C l(A)\right)$. Hence $A$ is $g_{X}^{i} g_{X}^{j}-$ closed.

2. ( $\Longrightarrow)$ Assume that $A$ is $g_{X}^{i} g_{X}^{j}-$ open. Thus $g^{i} \operatorname{Int}\left(g^{j} \operatorname{Int}(A)\right)=A$.

$g B d r_{i j}(A) \cap A=\left[g^{i} C l\left(g^{j} C l(A)\right) \backslash g^{i} \operatorname{Int}\left(g^{j} \operatorname{Int}(A)\right)\right] \cap A$

$$
\begin{aligned}
& =\left[g^{i} C l\left(g^{j} C l(A)\right) \backslash A\right] \cap A \\
& =\emptyset .
\end{aligned}
$$

Therefore, $g B d r_{i j}(A) \subseteq(X \backslash A)$.

$(\Longleftarrow)$ Assume that $g B d r_{i j}(A) \subseteq(X \backslash A)$. Thus $g B d r_{i j}(A) \cap A=\emptyset$, and also $\left[g^{i} C l\left(g^{j} C l(A)\right) \backslash g^{i} \operatorname{Int}\left(g^{j} \operatorname{Int}(A)\right)\right] \cap A=\emptyset$. Since $A \subseteq g^{i} C l\left(g^{j} C l(A)\right)$, we have $A \backslash g^{i} \operatorname{Int}\left(g^{j} \operatorname{Int}(A)\right)=\emptyset$. But $g^{i} \operatorname{Int}\left(g^{j} \operatorname{Int}(A)\right) \subseteq A$.

Consequently, $A=g^{i} \operatorname{Int}\left(g^{j} \operatorname{Int}(A)\right)$. Hence $A$ is $g_{X}^{i} g_{X}^{j}-$ open.

Theorem 3.6. Let $\left(X, g_{X}^{1}, g_{X}^{2}\right)$ be a bigeneralized topological space and $A$ be a subset of $X$. Then $g B d r_{i j}(A)=\emptyset$ if and only if $A$ is $g_{X}^{i} g_{X}^{j}-$ closed and $g_{X}^{i} g_{X}^{j}$-open where $i, j=1,2$ and $i \neq j$.

Proof. $(\Longrightarrow)$ Assume that $g B d r_{i j}(A)=\emptyset$.

Thus by Theorem 3.5, we have $A$ is $g_{X}^{i} g_{X}^{j}-$ closed and $g_{X}^{i} g_{X}^{j}-$ open.

$(\Longleftarrow)$ Assume that $A$ is $g_{X}^{i} g_{X}^{j}-$ closed and $g_{X}^{i} g_{X}^{j}-$ open.

By Theorem 3.5, we have $g B d r_{i j}(A) \subseteq A \cap(X \backslash A)=\emptyset$.

Hence, $g B d r_{i j}(A)=\emptyset$. 


\section{Acknowledgement}

The authors would like to thank Sakon Nakhon Rajabhat University for financial support.

\section{References}

[1] J.C. Kelly, Bitopological spaces, Pro. London Math. Soc., 313 (1963), 71-89.

[2] Á. Császár, Generalized topology, generalized continuity, Acta Math. Hungar, 96 (2002), 351-357.

[3] W.K. Min, Almost Continuity on Generalized Topological Spaces , Acta Math. Hungar, 125 (2009), 121-125.

[4] C. Boonpok, Weakly Open Functions on Bigeneralized Topological Spaces, Int. J. Math. Analysis, 4 (18) (2010), 891-897.

Received: August, 2012 